\title{
Virtual surgery simulation in orbital wall reconstruction: Integration of surgical navigation and stereolithographic models
}

\author{
Giorgio Novelli, Gabriele Tonellini, Fabio Mazzoleni, Alberto Bozzetti, Davide Sozzi \\ Department of Maxillo Facial Surgery, University of Milano - Bicocca, San Gerardo Hospital, Monza, Italy
}

\section{A R T I C L E I N F O}

\section{Article history:}

Paper received 16 April 2014

Accepted 25 September 2014

Available online 5 October 2014

\section{Keywords:}

Stereolithographic models

Surgical navigation

Orbital volume

Orbital wall reconstruction

Enophthalmos

\begin{abstract}
A B S T R A C $T$
Purpose: Correction of post traumatic orbital and zygomatic deformity is a challenge for maxillofacial surgeons. Integration of different technologies, such as software planning, surgical navigation and stereolithographic models, opens new horizons in terms of the surgeons' ability to tailor reconstruction to individual patients. The purpose of this study was to analyze surgical results, in order to verify the suitability, effectiveness and reproducibility of this new protocol.

Methods: Eleven patients were included in the study. Inclusion criteria were: unilateral orbital pathology; associated diplopia and enophthalmos or exophthalmos, and zygomatic deformities. Syndromic patients were excluded. Pre-surgical planning was performed with iPlan 3.0 CMF software and we used Vector Vision II (BrainLab, Feldkirchen, Germany) for surgical navigation. We used 1:1 skull stereolithographic models for all the patients. Orbital reconstructions were performed with a titanium orbital mesh. The results refer to: correction of the deformities, exophthalmos, enophthalmos and diplopia; correspondence between reconstruction mesh positioning and preoperative planning mirroring; and the difference between the reconstructed orbital volume and the healthy orbital volume.

Results: Correspondence between the post-operative reconstruction mesh position and the presurgical virtual planning has an average margin of error of less than $1.3 \mathrm{~mm}$. In terms of en- and exophthalmos corrections, we have always had an adequate clinical outcome with a significant change in the projection of the eyeball. In all cases treated, there was a complete resolution of diplopia. The calculation of orbital volume highlighted that the volume of the reconstructed orbit, in most cases, was equal to the healthy orbital volume, with a positive or negative variation of less than $1 \mathrm{~cm}^{3}$.

Conclusion: The proposed protocol incorporates all the latest technologies to plan the virtual reconstruction surgery in detail. The results obtained from our experience are very encouraging and lead us to pursue this path.
\end{abstract}

(C) 2014 European Association for Cranio-Maxillo-Facial Surgery. Published by Elsevier Ltd. All rights reserved.

\section{Introduction}

Correction of post traumatic orbital deformity and restoring orbital anatomy after tumor resection have long been a challenge for maxillofacial surgeons (Kawamoto, 1982; Ahn et al., 2008; Chen et al., 2006). Orbital shape and symmetry play an important functional and esthetic role in terms of projection and position of the eyeball, making corrective orbital three-dimensional anatomy reconstruction necessary. Orbital repair, however, is often made

\footnotetext{
* Corresponding author. Department of Maxillo Facial Surgery, University of Milano - Bicocca, San Gerardo Hospital, Via G.B. Pergolesi, 33, Monza (MB), 20900, Italy.

E-mail addresses: nove.gio@gmail.com, g.novelli@hsgerardo.org (G. Novelli).
}

difficult by variability in orbital conformation (Grant et al., 1997; Mathog et al., 1989).

Over the years, the development of reconstructive surgical techniques and technology has helped to improve surgical outcomes. Although surgical techniques are well defined, the problem relating to human error remains. The development of new materials and the advancement of technology have allowed us to address this problem, thus improving surgical outcomes (Schramm et al., 2009).

Technological development has covered four areas: image management and processing software, reconstruction materials, stereolithographic technology and surgical navigation. Presurgical planning has become possible through the development of image management software. Specifically, the use of stereolithographic models in maxillofacial surgery introduces the possibility of three- 
Table 1

Orbital pathology.

\begin{tabular}{ll}
\hline Disease & Number of patients \\
\hline $\begin{array}{l}\text { Outcome of blow-out orbital fracture, } \\
\text { floor and medial wall }\end{array}$ & 3 \\
$\begin{array}{l}\text { Outcome of blow-out orbital fracture, } \\
\quad \text { floor and medial wall + zygomatic fracture }\end{array}$ & 4 \\
Blow-out orbital fracture, floor and medial wall & 2 \\
Orbital bone tumor & 2 \\
\hline
\end{tabular}

dimensional presurgical planning directly on the model (Petzold et al., 1999). The introduction of preformed titanium meshes for orbital reconstruction makes it possible to manufacture custommade structures which facilitate the correct restoration of the orbital anatomy (Scolozzi et al., 2009; Scolozzi, 2011). Finally, the introduction of surgical navigation in cranio-maxillofacial surgery radically changed the surgical approach to facial and orbital diseases (Gellrich et al., 2002; Schmelzeisen et al., 2004; Jayaratne et al., 2010; Novelli et al., 2011). The advantage of navigation is that the surgeon can instantaneously determine the position of the surgical instrument on the CT images and see, during the operation, if the reconstruction is performed according to presurgical planning. In our department this technique is routinely used for orbital pathologies and mid-facial or orbital fractures (Novelli et al., 2012).

The integration of different technologies, particularly software, surgical navigation and stereolithography, opens new horizons for tailoring the reconstruction for each patient (Bell and Markiewicz, 2009; Tang et al., 2010; Zhang et al., 2010).

The purpose of this study was to analyze surgical results, in order to verify the suitability, effectiveness and reproducibility of this new protocol.

\section{Materials and methods}

Eleven patients, 9 male and 2 female, with an average age of 32 years (range 19-47) and different orbital pathologies were included in the study (Table 1). As inclusion criteria for the study, we considered the presence of unilateral orbital pathology, in association with diplopia and enophthalmos or exophthalmos, in non-syndromic patients (Table 2 ).

Presurgical planning was performed with iPlan 3.0 CMF software (BrainLab, Feldkirchen, Germany). For all the patients, we used 1:1 three dimensional stereolithographic models of the skull based on the DICOM data. Files were processed with 3Dyagnosys 4.0 software (3DIEMME, Italy). The STL model was printed by ZPrinter 310 (Z-Corporation, USA), a rapid prototyping machine, through an additive technique using deposition of chalk dust in

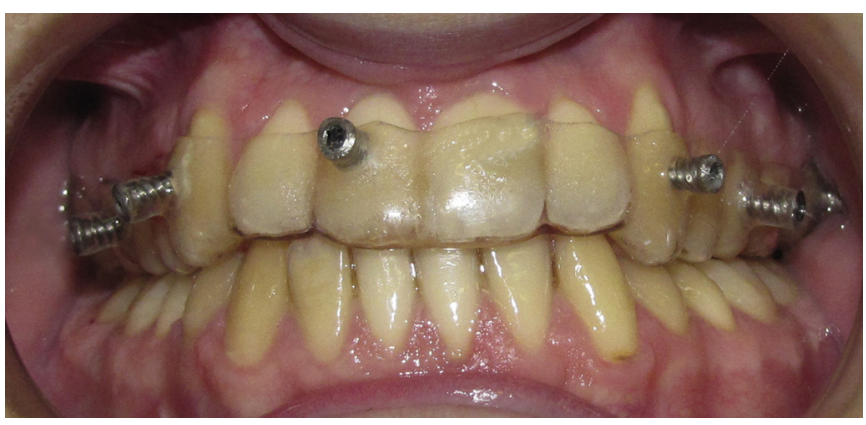

Fig. 1. Occlusal splint with five hexagonal-headed screws.

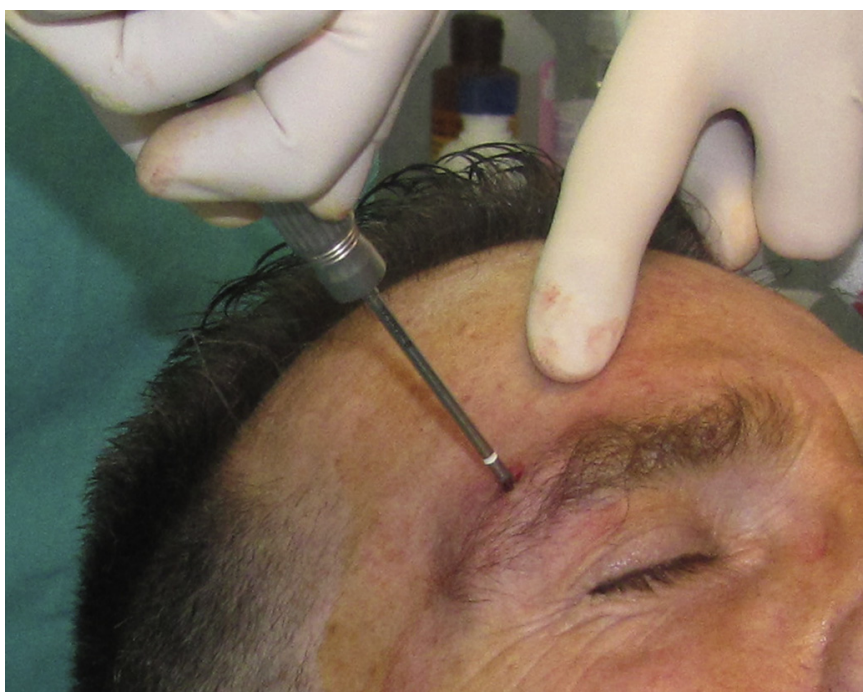

Fig. 2. Self-drilling screws fixed on the superior orbital frame.

$0.085 \mathrm{~mm}$ layers that was firmed up with water-based glue. The model obtained was subjected to infiltration with thermosetting resins and heat treatments to ensure mechanical resistance and the ability to withstand steam sterilization in an autoclave. Vector Vision II (BrainLab, Germany) was used for surgical navigation. In all the patients, orbital reconstructions were performed using a DePuySynthes (West Chester, PA, USA) preformed orbital mesh.

Our surgical reconstructive protocol consisted of eight steps:

Step one: DICOM data was captured with a maxillofacial CT scanner that produces $0.8-1 \mathrm{~mm}$ slices. The CT was acquired after positioning the patient's landmarks in order to orient the patient in space during surgical navigation. An occlusal splint anchored on the

Table 2

Inclusion criteria.

\begin{tabular}{|c|c|c|c|c|c|}
\hline & Diagnosis & Diplopia & $\begin{array}{l}\text { Exophthalmos } \\
\mathrm{mm}\end{array}$ & $\begin{array}{l}\text { Enophthalmos } \\
\mathrm{mm}\end{array}$ & $\begin{array}{l}\text { Craniomaxillofacial } \\
\text { malformations }\end{array}$ \\
\hline 1 & Blow-out orbital fracture, floor and medial wall & $\mathbf{X}$ & & 3.9 & No \\
\hline 2 & Results of blow-out orbital floor fracture & $\mathbf{X}$ & & 4.3 & No \\
\hline 3 & Results of blow-out orbital fracture, floor and medial wall & $\mathbf{X}$ & & 0 & No \\
\hline 4 & Blow-out orbital fracture, floor and medial wall & $\mathbf{X}$ & & 3.2 & No \\
\hline 5 & Results of blow-out orbital fracture, floor and medial wall + zygomatic fracture & $\mathbf{X}$ & & 3.1 & No \\
\hline 6 & Blow-out orbital fracture floor and medial wall & $\mathbf{X}$ & & 3.1 & No \\
\hline 7 & Results of blow-out orbital fracture, floor and medial wall + zygomatic fracture & $\mathbf{X}$ & & 3.5 & No \\
\hline 8 & Results of blow-out orbital fracture, floor and medial wall + zygomatic fracture & $\mathbf{X}$ & & 3 & No \\
\hline 9 & Results of blow-out orbital fracture, floor and medial wall + zygomatic fracture & Vision loss & & 6 & No \\
\hline 10 & Orbital bone tumor (osteoblastoma) & $\mathbf{X}$ & 2 & & No \\
\hline 11 & Orbital bone tumor (ossifying fibroma) & $\mathbf{X}$ & 2 & & No \\
\hline
\end{tabular}

$\mathrm{X}$ represents "yes". 


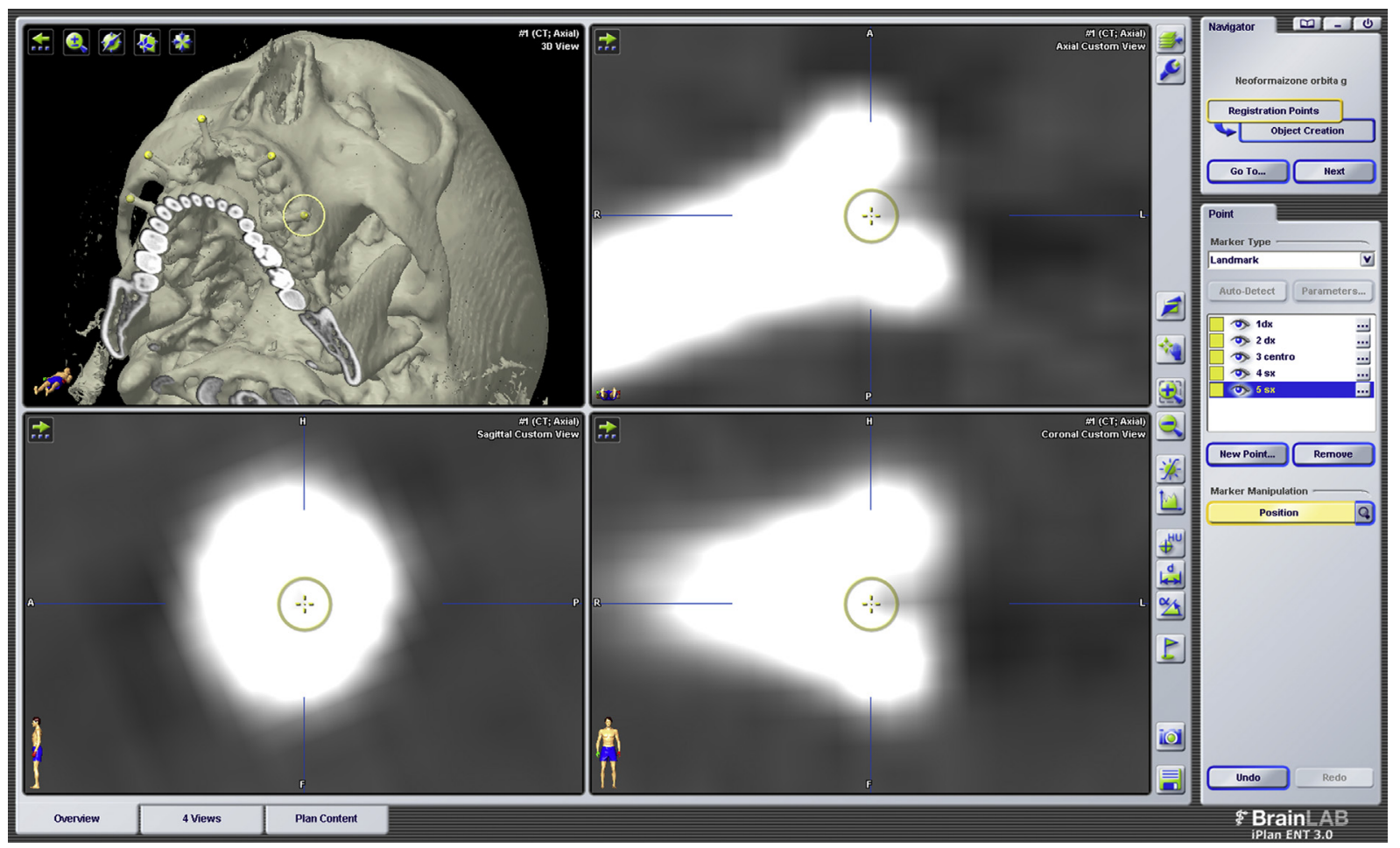

Fig. 3. Identification of the landmarks.

maxilla with five hexagonal-headed screws was used (Fig. 1). To improve the accuracy of registration in all the patients, we combined the splint with two orbital bone markers represented by bilateral self-drilling screws fixed on the superior orbital frame (Novelli et al., 2012) (Fig. 2).

Step two: DICOM data was processed with iPlan 3.0 CMF software. After determining the planes of symmetry on the CT data, which is necessary for mirroring, the landmarks were identified. Reference points on the CT must be easily identifiable during the registration process (Fig. 3). Presurgical planning proceeded with the anatomical identification of the unaffected side using the auto segmentation function. Furthermore, the mirroring of the non-affected to the affected side was performed using a virtual mid-sagittal plane (Fig. 4). The software is not only a programming tool but also a diagnostic tool since it provides information about the size of the defects and possible strategies to correct them.

Step three: The stereolithographic model was manufactured by exporting the patient's STL file of the skull and of the maxillofacial regions. The stereolithographic model can be planned and constructed with two methods: exactly reproducing the patient's anatomy with a 1:1 ratio or mirroring the non-affected side, thus obtaining an anatomical model without the disease. In some cases, such as in the presence of an orbital tumor, we can virtually remove the pathological tissue, mirror the outer surface of the orbital bone from the non-affected to the affected side and finally export the STL file to generate the stereolithographic model (Fig. 5).

Step four: This involved acquiring the reference points, which makes the registration and navigation of the model possible. This step is needed in order to perform stereolithographic model navigation, since it is not possible to apply the patient's occlusal bite. Tooth enamel CT has a different density compared with bone. This leads to an artifact on the model which prevents bite positioning. There must be at least four landmarks and we usually use the point of origin of the infraorbital nerve and the zygomatic nerve, which are easily detectable on the model and on the CT images (Fig. 6).

An alternative registration technique of the stereolithographic model is laser surface scanning. One limitation of this method is the transparency of the model, which does not reflect the laser light. In

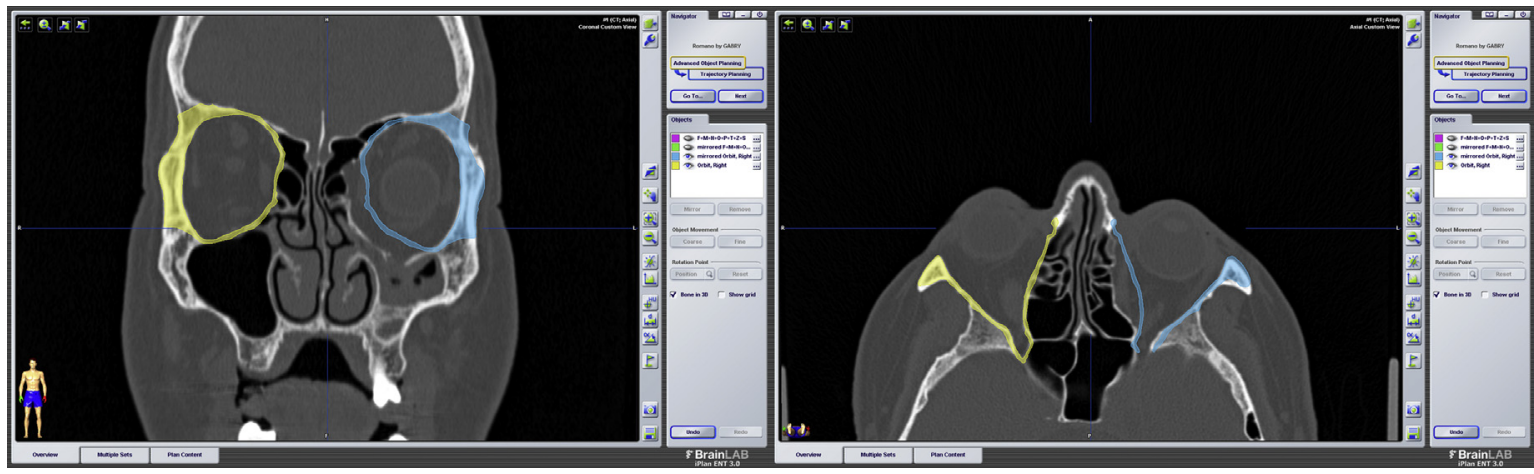

Fig. 4. Mirroring of the non-affected side to the affected side. 


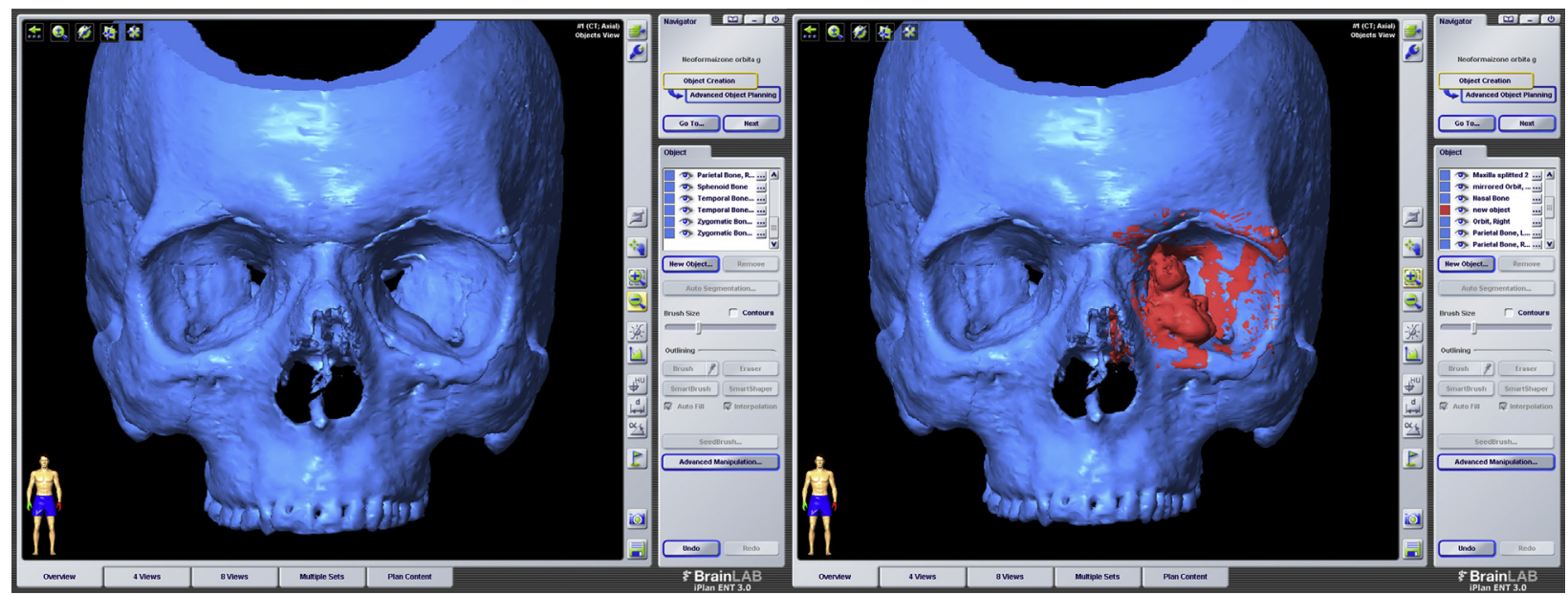

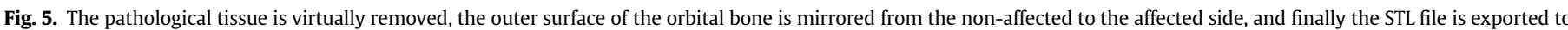
generate the stereolithographic model.

cases where healthy bone is well represented on the model, we can use the bone surface detection point method. The recording of the model is based on a dynamic reference frame (DRF) which is fixed on the model itself (Fig. 7).

Once the registration process is completed, one can browse the model and verify accuracy.

Step five: The preparation of a titanium mesh that will be used for orbital reconstruction (Fig. 8). During this phase preformed plates are generally used, which have the advantage of reproducing the shape of the floor and of the lateral orbital wall. Although the plates are preformed, minor modeling is possible in order to improve the fit of the plate on the stereolithographic model. In some case where large reconstruction is required, we mold a titanium mesh, thus customizing the surgical planning directly on the model. Obviously in large reconstructions using multiple meshes these will need to be separated to make surgical access and positioning possible. Subsequently, the shape and the reconstruction are checked under navigation control.

Step six: Acquiring the virtual position of the titanium mesh The mesh was fixed with screws to the model and the screw



Fig. 6. Acquiring the reference points, which make the registration and navigation of the model possible. 


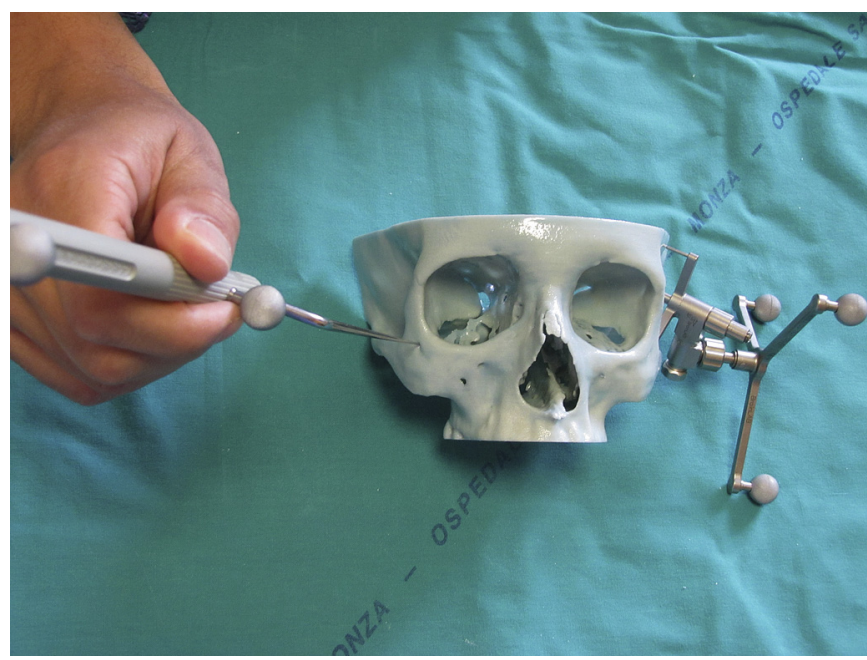

Fig. 7. Model registration on the patient's CT scan.

position was acquired. At this stage all the necessary points to reproduce the correct surgical orientation of the titanium mesh were imported (Fig. 9).

Step seven: The surgery itself. In all cases a transconjunctival access with retrocaruncular extension was used. In two cases of orbito-zygomatic fracture it was necessary to reposition the zygoma. In those cases a lateral canthotomy was added to the transconjunctival access, as well as an intraoral and hemicoronal access in order to perform osteotomies and to correctly reposition the body and the zygomatic arch. In reconstructive cases the navigator was useful for identifying bone surfaces while in cases of orbital tumor, navigation facilitates the resection step.

After preparation of the surgical site, the mesh stabilization screws are positioned as previously planned (Fig. 10). The next step was to check, through surgical navigation, whether the reconstruction has followed the presurgical plan (Fig. 11).

Step eight: The postoperative check with CT scan. After acquiring the scans, we fused the pre-and post-surgical CT scans. The overlap of the mirroring on the postoperative CT scan could be used to check the adequacy of the reconstruction performed (Fig. 12). A postoperative CT scan could be done immediately after the surgery in the operating theater.

To assess data, we analyzed clinical and radiographic parameters such as:

1. Correction of en- or exophthalmos measured in millimeters on the presurgical and postoperative CT scans. Evaluation of the eyeball position was done by linear measurements using CT scan images. We used the Cabanis index (Cabanis et al., 1980; Doyon et al., 1990). Before acquiring the measurements it is essential to align the skull on the Frankfort and sagittal planes (Fig. 13). After alignment on the axial view, the bicantal external plane (BCEP) was drawn. Subsequently a perpendicular line to the BCPE was drawn between corneal surface point and BCPE (the anterior bicantal external segment - ABCES) (Fig. 14).

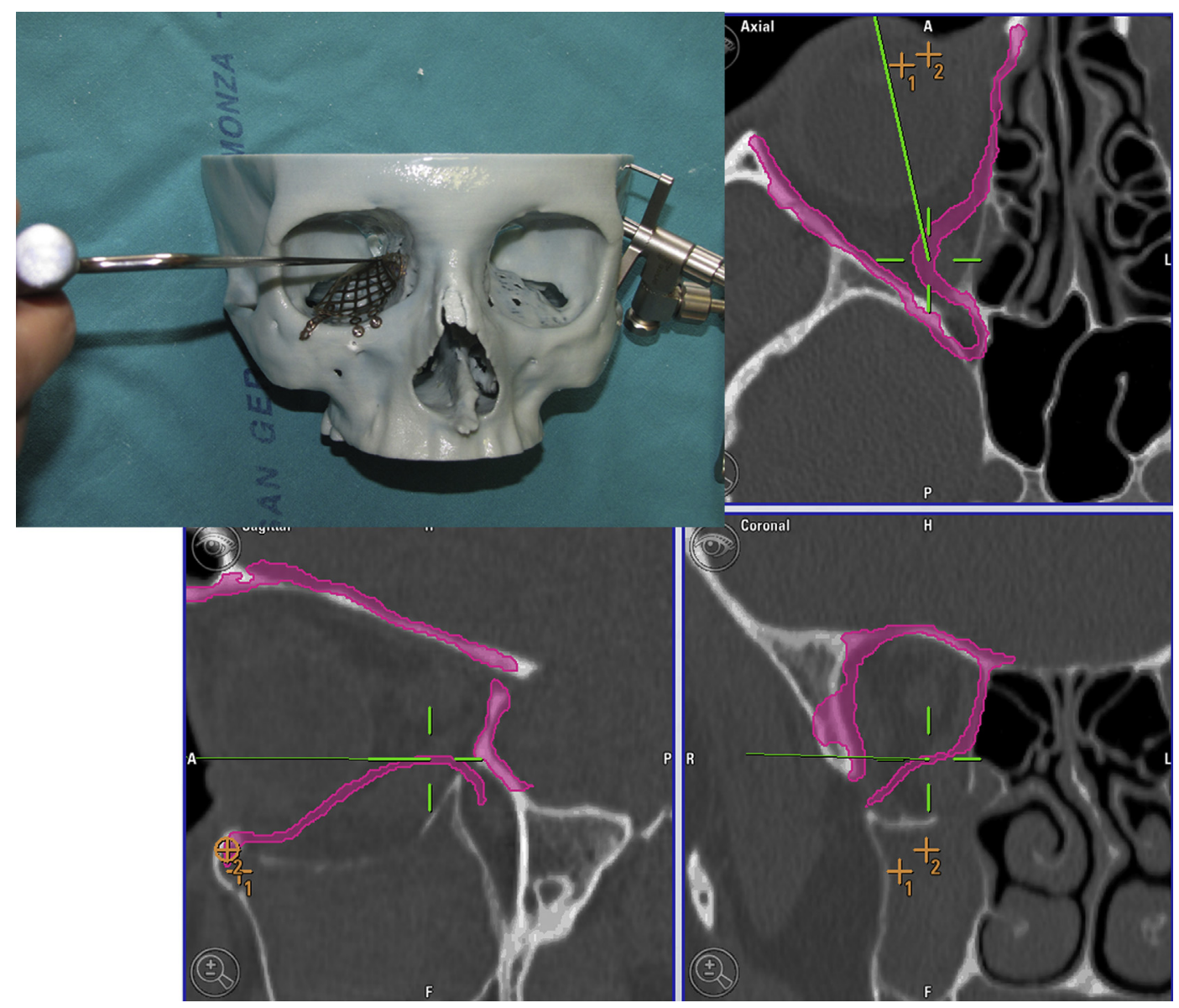

Fig. 8. Check the mesh position according to the planning. 

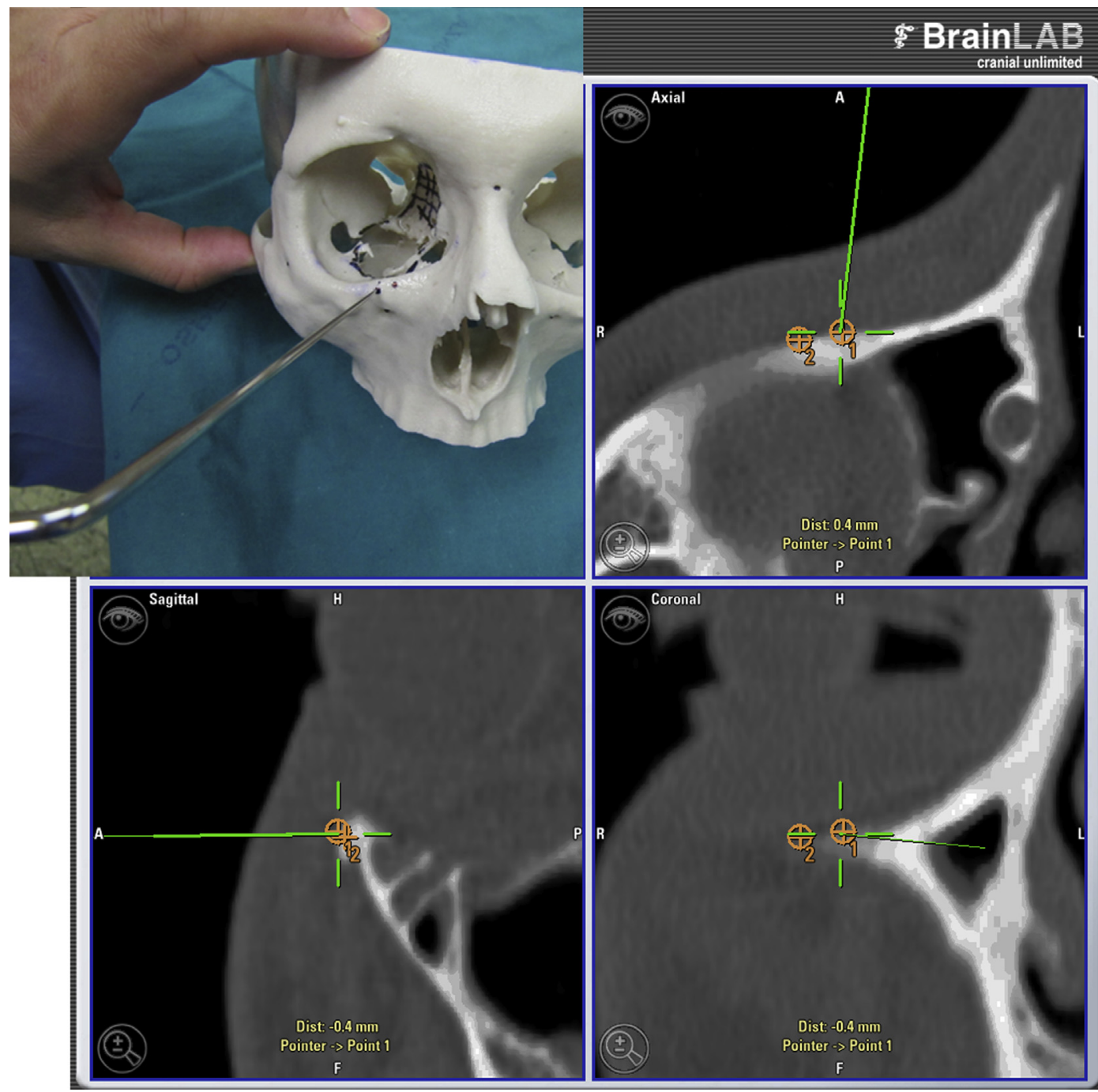

Fig. 9. Acquire all the necessary points to reproduce the correct surgical orientation of the titanium mesh.

This technique, unlike the classical methods such as Hertel's exophthalmometry, allows for accurate calculation of the position of the eyeball, eliminating possible interference related to edema in cases of trauma or modification of the hard and soft tissues as in tumor cases or trauma sequelae. In cases of orbital- zygomatic fracture, mirroring was used for the evaluation of the eyeball position.

2. Comparison of the reconstructed orbital volume to that of the healthy orbit. The volumetric analysis of the orbits was performed by using the software iPlan3.0 CMF. In the literature

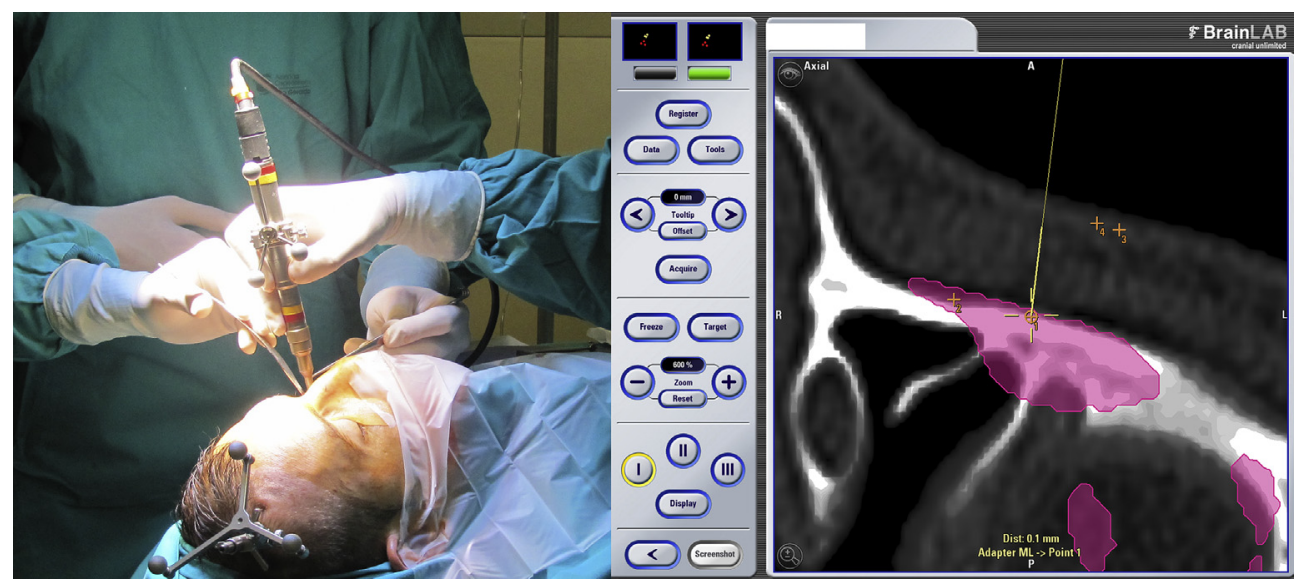

Fig. 10. Location of the mesh stabilization screws with navigation assistance, and reproduction of the mesh position. 


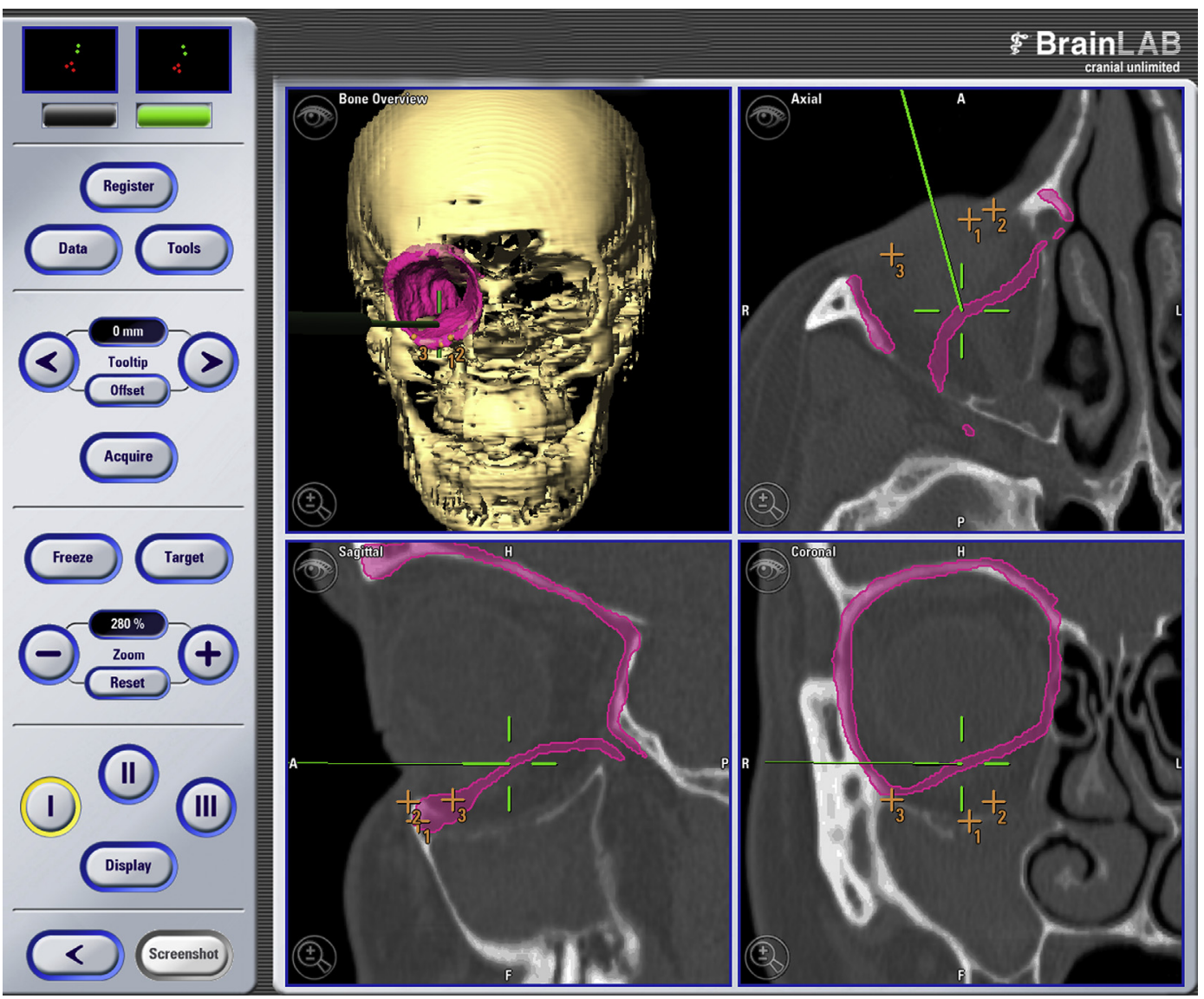

Fig. 11. Intraoperative mesh positioning with real time navigation imaging.

there has been an attempt to calculate the orbital volume using different software. The limitation of these methodologies is the manual definition of both the front and rear limits of orbital volume by the surgeon or technician (Bite et al., 1985; Carls et al., 1996; Chan et al., 2000; Deveci et al., 2000; Fan et al. 2003; Kwon et al., 2009, 2010; Ozyazgan et al., 2009; Raskin et al., 1998; Ramieri et al., 2000; Regensburg et al., 2008, 2011; Scolozzi and Jaques, 2008; Tahernia et al., 2009).

The autosegmentation process of the orbital structures and the orbital volume allows for automatic calculation through an algorithm which is characteristic of the software. The front and rear orbital limits are defined by the software, to avoid possible operator-dependent errors (Fig. 15).

3. Correction of diplopia evaluated with preoperative and postoperative Hess-Lancaster test.

4. Overlap of the pre-surgical plan on the post-operative CT scan, considering the discrepancy in coronal and sagittal sections in millimeters. We evaluated the correspondence, in millimeters, between the virtual reconstruction and surgical result. To validate the data, we superimposed the virtual 3D reconstruction on the pre-op CT scan with the post-operative CT scan through image fusion.

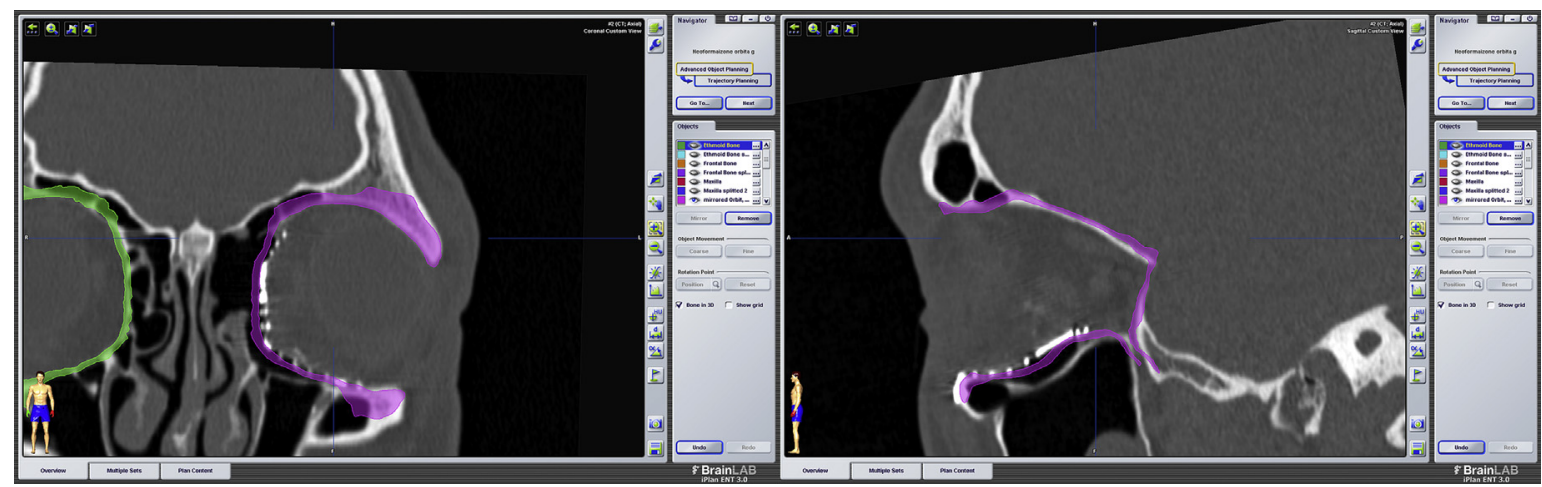

Fig. 12. Postoperative $\mathrm{CT}$ scan with image superimposition showing the perfect match between mirroring and mesh positioning. 


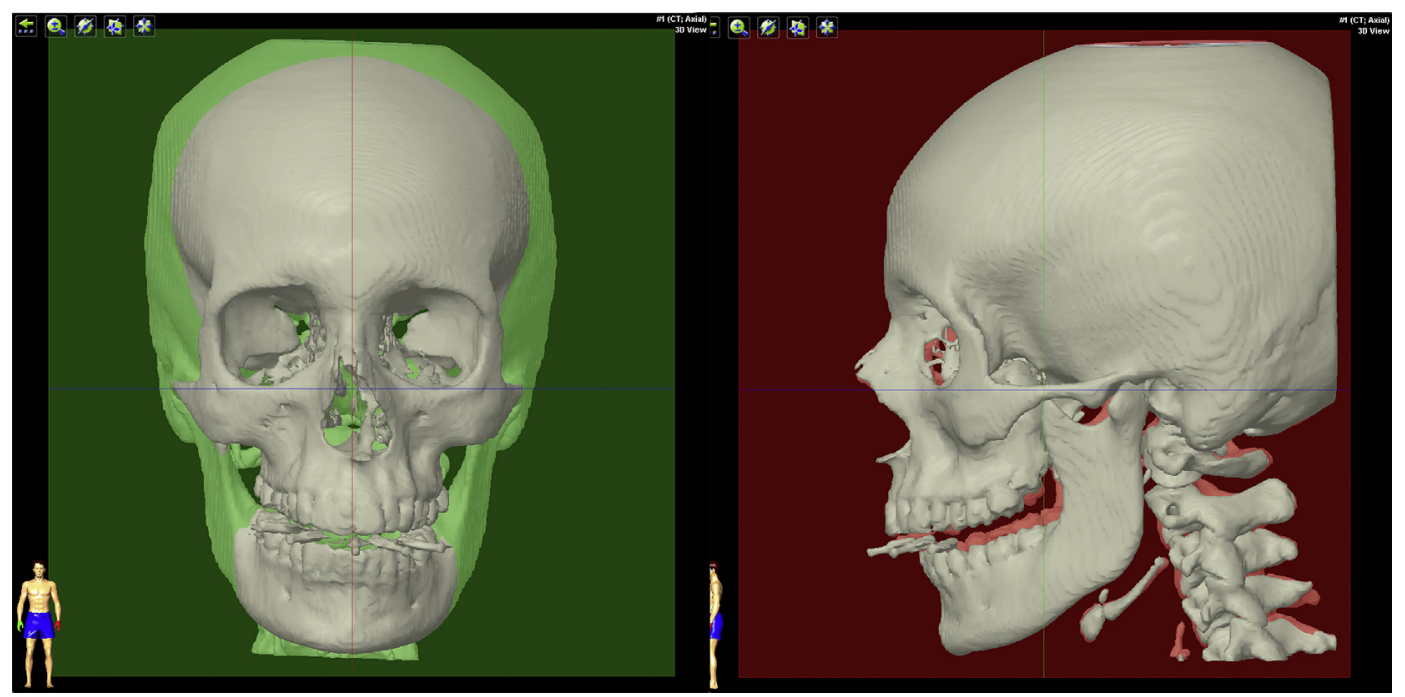

Fig. 13. Align the skull on the sagittal plane and the Frankfort plane.

\section{Results}

The data obtained from analysis of the 11 patients are reported in Table 3. The results show that the correspondence between postoperative reconstruction mesh position and presurgical virtual planning has an average margin of error of less than $1.3 \mathrm{~mm}$. These data confirmed that the reconstruction performed faithfully reproduced the planned shape of the orbital walls. In terms of enand exophthalmos correction, we have always had an adequate clinical outcome with a significant change in the projection of the eyeball. In all the cases treated, there was a complete resolution of diplopia.

Evaluation of the orbital reconstruction was performed by comparing the values of the healthy orbital volume and the reconstructed orbital volume in each patient; Table 4 shows the interesting data. The calculation of orbital volume highlighted that in most cases when the reconstructed orbits were superimposed on the healthy orbital volume, there was a positive or negative variation of less than $1 \mathrm{~cm}^{3}$. The results confirm that the anatomical reconstruction of the planned orbital walls corresponds to an adequate orbital volume restoration.

In one case, the post-operative volume was $2.2 \mathrm{~cm}^{3}$ less than the contralateral side. This was a case of orbital osteoblastoma where

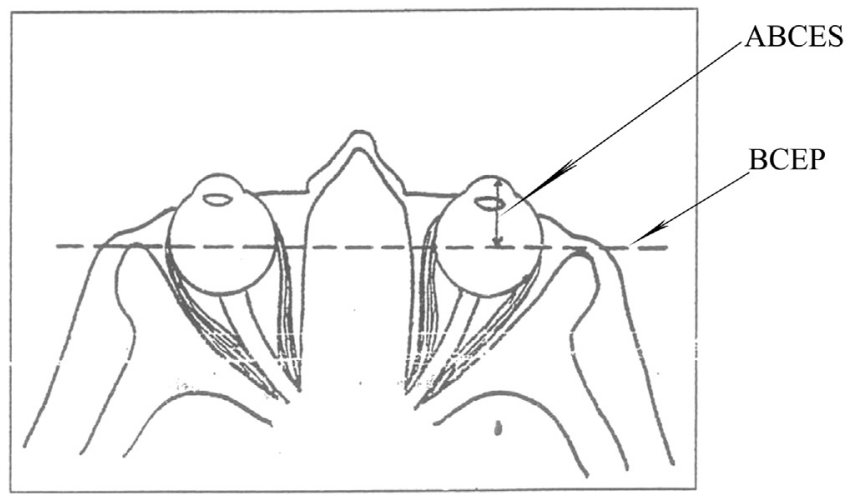

Fig. 14. Cabanis index: the bicantal external plane (BCEP) is drawn. Subsequently a perpendicular line at the BCPE is drawn between corneal surface point and BCPE (anterior bicantal external segment - ABCES) (Cabanis et al., 1980; Doyon et al., 1990). the tumor had partially modified the anatomy of the orbital walls. Furthermore, the position of the reconstruction mesh, while being in-line with what was planned, produces a thicker neo wall than mirroring does. This resulted in an orbital volume calculation by the software which was lower than the contralateral side, allowing a slight overcorrection in favor of the final esthetic and functional result.

\section{Discussion}

The orbit is shaped like a quadrangular pyramid with the base anteriorly positioned, an apex, a trunk and a rear. This implies that the volume of the posterior third is relatively small. Therefore, a volume reduction or a volume increase of this region may cause enophthalmos or exophthalmos, diplopia and sometimes dystopia of the eyeball. The orbital walls have a unique anatomical conformation. The orbital floor is concave in the anterior two-thirds and convex in the posterior third, like an italic $S$ in the sagittal plane. The medial wall, represented by the perpendicular ethmoid lamina, is convex.

Proper reconstruction of the orbital walls plays an important role in restoring accurate vision and the orbital region's symmetry, thus ensuring function as well as aesthetics. The surgeon's goal has always been to obtain the best orbital wall reconstruction with the most suitable materials, with the final result dependent on their own skill. Different surgical techniques have been proposed to correct the position of the eyeball or reconstruct the orbital walls. It has never been possible to adequately reconstruct the exact anatomy of the orbital cavity.

The critical points in orbital reconstruction are:

a) The orbital floor: the most anterior portion gives the vertical position of the eyeball and the rear portion, where the orbital floor articulates with the greater wing of the sphenoid, determines the projection of the eyeball.

b) The medial wall of the orbit, represented by the ethmoid, is often very convex especially posteriorly. Furthermore this anatomical area contributes to the projection of the eyeball.

In the last fifteen years, the evolution of imaging and hardware technology with new generation materials has brought a significant benefit, making the surgeon's job easier. Nevertheless, it is with the 


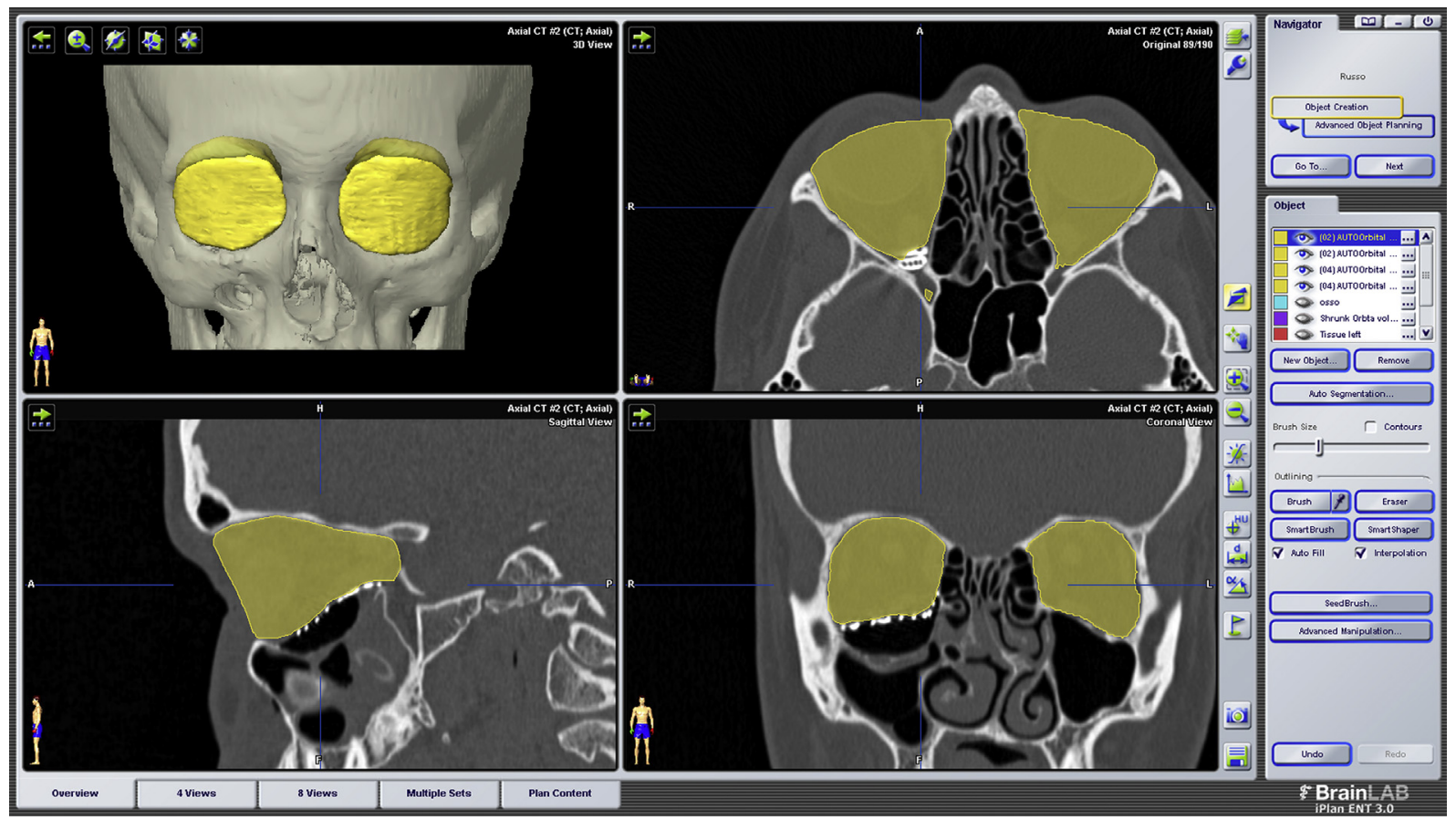

Fig. 15. Volumetric analysis of the orbits using the software iPlan3.0 CMF.

Table 3

Results in terms of enophthalmos or exophthalmos and diplopia correction, and evaluation of the mismatch between mirroring and reconstruction by using CT scan superimposition.

\begin{tabular}{llllll}
\hline & $\begin{array}{l}\text { Enophthalmos } \\
\text { pre }(\mathrm{mm})\end{array}$ & $\begin{array}{l}\text { Enophthalmos } \\
\text { post }(\mathrm{mm})\end{array}$ & $\begin{array}{l}\text { Diplopia } \\
\text { pre }\end{array}$ & $\begin{array}{l}\text { Diplopia } \\
\text { post }\end{array}$ & $\begin{array}{l}\text { Mismatch in mm } \\
\text { between mirroring } \\
\text { and reconstruction }\end{array}$ \\
\hline 1 & 3.9 & 0.4 & Yes & No & $\leq 2$ \\
2 & 4.3 & 0.2 & Yes & No & $\leq 1$ \\
3 & 0 & 0 & Yes & No & $\leq 1$ \\
4 & 3.2 & 0 & Yes & No & $\leq 1$ \\
5 & 3.1 & 0 & Yes & No & $\leq 2$ \\
6 & 3.1 & 0 & Yes & No & $\leq 2$ \\
7 & 3.5 & 0.5 & Yes & No & $\leq 2$ \\
8 & 3 & 0 & Yes & No & $\leq 1$ \\
9 & 6 & 0.8 & Vision & - & $\leq 1$ \\
& & & loss & & \\
& & & & & \\
& Exophthalmos & Exophthalmos & & & \\
\hline 10 & 2 & post (mm) & & Yes & No \\
11 & 2 & 0 & No & No & $\leq 1$ \\
& & 0 & Total average & $\mathbf{1 . 3}$ \\
\hline
\end{tabular}

Table 4

Evaluation of the difference between the reconstructed orbital volume and the healthy orbital volume using the software iPlan3.0 CMF.

\begin{tabular}{llll}
\hline & $\begin{array}{l}\text { Reconstructed orbital } \\
\text { volume }\left(\mathrm{cm}^{3}\right)\end{array}$ & $\begin{array}{l}\text { Healthy orbital } \\
\text { volume }\left(\mathrm{cm}^{3}\right)\end{array}$ & $\Delta\left(\mathrm{cm}^{3}\right)$ \\
\hline 1 & 30.956 & 30.517 & +0.4 \\
2 & 32.012 & 32.369 & -0.357 \\
3 & 29.749 & 29.910 & -0.16 \\
4 & 30.122 & 31.109 & -0.987 \\
5 & 30.216 & 29.190 & +1.02 \\
6 & 27.673 & 27.839 & -0.166 \\
7 & 31.558 & 30.997 & +0.561 \\
8 & 31.045 & 30.998 & +0.047 \\
9 & 32.889 & 31.050 & -1.839 \\
10 & 34.935 & 37.158 & -2.2 \\
11 & 34.807 & 35.243 & -0.436 \\
& & Total average & $-\mathbf{0 . 2 1}$ \\
\hline
\end{tabular}

introduction of surgical navigation in maxillofacial surgery, that there has been a turning point in the reconstruction of the orbit. The integration of software planning, surgical navigation and customization offers a real and undisputed advantage. Presurgical planning based on how the mesh bends on the stereolithographic model to repair the orbital fracture is not recent (Bell and Markiewicz, 2009; Klug et al., 2006).

The innovation of our protocol is the ability to accurately perform surgical navigation on the stereolithographic model. This makes it possible to virtualize the surgery and then reproduce it in the operating room. Our protocol can be integrated with CAD-CAM patient-specific implant modeling, this method offers great possibilities but increases the costs for the hospital. The advantages of the intraoperative CT scan are: direct control of the results in the operating theater and the immediate correction of any errors.

Unique to our method is the ability to place the three-dimensional mesh as programmed, with a margin of error of less than $1 \mathrm{~mm}$. Data analysis shows that the results are extremely predictable.

\section{Conclusion}

The proposed protocol incorporates all the latest technology to plan virtual reconstruction surgery in detail. The advantages for the surgeon are significant. First, during the presurgical stage, this is an important tool for diagnosis and planning, giving a better understanding of how the defect can be reconstructed.

As a second advantage, the surgeon can verify the reconstruction of every millimeter of the mesh, and validate the correspondence between the virtual plan and the mesh on the stereolithographic model. The third advantage is that this makes the surgical reconstruction easier, dramatically reducing surgical time. Finally, it is possible to standardize the planning phases making the protocol reproducible and adaptable to different clinical cases.

The disadvantage of this procedure is the time spent in presurgical planning. In accordance with the most recent literature, this protocol considerably reduces the need for any reinterventions. There is also a reduction in biological costs for 
patients and a decrease in healthcare costs. The results obtained from our experience are very encouraging. Extending the use of this protocol to other clinical-pathological frames will enlarge the cohort of patients confirming the validity of the protocol.

\section{Disclosure}

None of the authors has a financial interest in any of the products, devices, or drugs mentioned in this article.

\section{References}

Ahn HB, Ryu WY, Yoo KW, Park WC, Rho SH, Lee JH, et al: Prediction of enophthalmos by computer-based volume measurement of orbital fractures in a Korean population. Ophthal Plast Reconstr Surg 24(1): 36-39, 2008

Bell RB, Markiewicz MR: Computer-assisted planning, stereolithographic modeling, and intraoperative navigation for complex orbital reconstruction: a descriptive study in a preliminary cohort. J Oral Maxillofac Surg 67(12): 2559-2570, 2009

Bite U, Jackson IT, Forbes GS, Gehring DG: Orbital volume measurements in enophthalmos using three-dimensional CT Imaging. Plast Reconst Surg 75(4): $502-508,1985$

Cabanis EA, Haut J, Iba-Zizen MT, Palacin JJ, Porret G, Laplaud O, et al: Exophthalmometrie tomodensitometrique et biometrie T.D.M: oculo-orbitarie. Bull Soc Opht France 80: 63-66, 1980

Carls FR, Josca R, Sailer HF: The measurement of orbital volume in reconstruction of the orbital walls. Minerva Stomatol 45(11): 493-499, 1996

Chan CH, Spalton DJ, McGurk M: Quantitative volume replacement in the correction of post-traumatic enophthalmos. Br J Oral Maxillofac Surg 38(5): 437-440, 2000

Chen CT, Huang F, Chen YR: Management of posttraumatic enophthalmos. Chang Gung Med J 29(3): 251-261, 2006

Deveci M, Oztürk S, Sengezer M, Pabușcu Y: Measurement of orbital volume by a 3 dimensional software program: an experimental study. J Oral Maxillofac Surg 58(6): 645-648, 2000

Doyon D, Laval-Jentet M, Halimi PH, Cabanis EA, Frija J: Orbite in Tomografia computerizzata. Italy: Masson, 1990

Fan X, Li J, Zhu J, Li H, Zhang D: Computer-assisted orbital volume measurement in the surgical correction of late enophthalmos caused by blowout fractures. Ophthal Plast Reconstr Surg 19(3): 207-211, 2003

Gellrich NC, Schramm A, Hammer B, Rojas S, Cufi D, Lagrèze W, et al: Computerassisted secondary reconstruction of unilateral posttraumatic orbital deformity. Plast Reconstr Surg 110(6): 1417-1429, 2002

Grant MP, Iliff NT, Manson PN: Strategies for the treatment of enophthalmos. Clin Plast Surg 24(3): 539-550, 1997

Jayaratne YS, Zwahlen RA, Lo J, Tam SC, Cheung LK: Computer-aided maxillofacial surgery: an update. Surg Innov 17(3): 217-225, 2010

Kawamoto HK: Late posttraumatic enophthalmos: a correctable deformity? Plast Reconstr Surg 69(3): 423-432, 1982

Klug C, Schicho K, Ploder O, Yerit K, Watzinger F, Ewers R, et al: Point-to-point computer-assisted navigation for precise transfer of planned zygoma osteotomies from the stereolithographic model into reality. J Oral Maxillofac Surg 64(3): 550-559, 2006

Kwon J, Barrera JE, Jung TY, Most SP: Measurements of orbital volume change using computed tomography in isolated orbital blowout fractures. Arch Facial Plast Surg 11(6): 395-398, 2009
Kwon J, Barrera JE, Most SP: Comparative computation of orbital volume from axia and coronal CT using three-dimensional image analysis. Ophthal Plast Reconstr Surg 26(1): 26-29, 2010

Mathog RH, Hillstrom RP, Nesi FA: Surgical correction of enophthalmos and diplopia. Arch Otolaryngol Head Neck Surg 115(2): 169-178, 1989

Novelli G, Ferrari L, Sozzi D, Mazzoleni F, Bozzetti A: Transconjunctival approach in orbital traumatology: a review of 56 cases. J Craniomaxillofac Surg 39(4): 266-270, 2011

Novelli G, Tonellini G, Mazzoleni F, Sozzi D, Bozzetti A: Surgical navigation recording systems in orbitozygomatic traumatology. J Craniofac Surg 23(3): 890-892, 2012

Ozyazgan I, Erdoğan N, Sahin B: Stereologic orbital volume measurements in zygomatic fractures. J Oral Maxillofac Surg 67(12): 2605-2608, 2009

Petzold R, Zeilhofer HF, Kalender WA: Rapid protyping technology in medicine-basics and applications. Comput Med Imaging Graph 23(5): 277-284 1999

Ramieri G, Spada MC, Bianchi SD, Berrone S: Dimensions and volumes of the orbit and orbital fat in posttraumatic enophthalmos. Dentomaxillofac Radiol 29(5): 302-311, 2000

Raskin EM, Millman AL, Lubkin V, DellaRocca RC, Lisman RD, Maher EA: Prediction of late enophthalmos by volumetric analysis of orbital fractures. Ophthal Plast Reconstr Surg 14(1): 19-26, 1998

Regensburg NI, Kok PH, Zonneveld FW, Baldeschi L, Saeed P, Wiersinga WM, et al: A new and validated CT-based method for the calculation of orbital soft tissue volumes. Invest Ophthalmol Vis Sci 49(5): 1758-1762, 2008

Regensburg NI, Wiersinga WM, van Velthoven ME, Berendschot TT, Zonneveld FW, Baldeschi L, et al: Age and gender-specific reference values of orbital fat and muscle volumes in Caucasians. Br J Ophthalmol 95(12): 1660-1663, 2011

Schmelzeisen R, Gellrich NC, Schoen R, Gutwald R, Zizelmann C, Schramm A: Navigation-aided reconstruction of medial orbital wall and floor contour in cranio-maxillofacial reconstruction. Injury 35(10): 955-962, 2004

Schramm A, Suarez-Cunqueiro MM, Rücker M, Kokemueller H, Bormann KH Metzger MC, et al: Computer-assisted therapy in orbital and mid-facial reconstructions. Int J Med Robot 5(2): 111-124, 2009

Scolozzi P: Reconstruction of severe medial orbital wall fractures using titanium mesh plates placed using transcaruncular-transconjunctival approach: a successful combination of two techniques. J Oral Maxillofac Surg 69(5): 1415-1420, 2011

Scolozzi P, Jaques B: Computer-aided volume measurement of posttraumatic orbits reconstructed with AO titanium mesh plates: accuracy and reliability. Ophthal Plast Reconstr Surg 24(5): 383-389, 2008

Scolozzi P, Momjian A, Heuberger J, Andersen E, Broome M, Terzic A, et al: Accuracy and predictability in use of AO three-dimensionally preformed titanium mesh plates for posttraumatic orbital reconstruction: a pilot study. J Craniofac Surg 20(4): 1108-1113, 2009

Tahernia A, Erdmann D, Follmar K, Mukundan S, Grimes J, Marcus JR: Clinical implications of orbital volume change in the management of isolated and zygomatico-maxillary complex-associated orbital floor injuries. Plast Reconstr Surg 123(3): 968-975, 2009

Tang W, Guo L, Long J, Wang H, Lin Y, Liu L, et al: Individual design and rapid prototyping in reconstruction of orbital wall defects. J Oral Maxillofac Surg 68(3): 562, 2010

Zhang Y, He Y, Zhang ZY, An JG: Evaluation of the application of computer-aided shape-adapted fabricated titanium mesh for mirroring-reconstructing orbital walls in cases of late post-traumatic enophthalmos. J Oral Maxillofac Surg 68(9): 2070-2075, 2010 